\title{
El dispositivo TUYA como estrategia de personalización en educación en línea
}

\author{
Mariángeles Castro Sánchez \\ Instituto de Ciencias para la Familia, Universidad Austral. \\ Mcastrosanchez@austral.edu.ar \\ ORCID: https://orcid.org/0000-0001-6874-668 \\ Fecha de finalización: 31 de agosto de 2021. \\ Recibido: 31 de agosto de 2021 \\ Aceptado: 28 de octubre de 2021. \\ DOI: https://doi.org/10.26422/aucom.2021.1002.cas
}

\section{Resumen}

El informe condensa la experiencia 2021 de la asignatura Familia y Tecnología, que integra el currículo de la Licenciatura en Orientación Familiar de la Universidad Austral de Argentina, en el tercer año de la carrera. Se trata de un espacio a distancia, desarrollado en el campus virtual de la universidad en la plataforma Moodle 3.9, con una carga horaria intensiva de 60 horas a lo largo de un mes calendario.

Como centro de la propuesta se despliega el dispositivo TUYA (trayectorias ubicuas y autorreguladas), que compone un planteo novedoso, disruptivo y contrastante con los abordajes usuales de los espacios educativos en línea. El objetivo es promover la autorregulación de los estudiantes a través de una aproximación al objeto de estudio y a los contenidos específicos de la asignatura basada en sus intereses particulares, en la que la autogestión del tiempo se presenta como un elemento nuclear. Esto se concreta a partir de un acceso ubicuo no solo del estudiantado al campus, sino de los participantes inmersos en la propia dinámica del aula virtual.

El resultado de la experiencia se traduce en aprendizajes significativos, tanto para estudiantes como para docentes, cuyas evidencias son las producciones, pero también las marcas de los procesos reflexivos promovidos durante el recorrido y profundizados en el momento de cierre de la cursada.

Palabras clave: educación en línea, aprendizaje ubicuo, personalización, autorregulación, educación basada en competencias.

\section{The TUYA approach as a personalization strategy for online education Abstract}

This report is a summary of the experiences and insights gathered across 2021 within the Family and Technology course at Austral University in Argentina. Students take this course during the third year of their Family Counseling bachelor's degree program. Attendance is carried out remotely, through the university's virtual campus in the Moodle 3.9 learning platform. Course load is 60 hours a month.

At the center of it all is the TUYA approach, a Spanish acronym meaning: "Ubiquitous and self-regulated trajectories." This approach is novel and disruptive, countering the typical alterna- 
Mariángeles Castro Sánchez

El dispositivo TUYA como estrategia de personalización en educación en línea

tives usually found in online educational spaces. The goal is to promote students' self-regulation skills, bringing them closer to the subject matter and the course's specific contents by considering their own, particular interests. Students' own time-management is one of the central tenets of this approach. And to make this possible, students are granted ubiquitous access, both to the campus and to the dynamics of the virtual classroom itself.

This experience has led to important insights, both for students and faculty. These can be derived from students' own coursework as well as the thinking processes promoted during the course and reinforced at the very end.

Keywords: online education, ubiquitous learning, personalization, self-regulation, skill-based education.

\section{O dispositivo TUYA como estratégia de personalização na educação online}

Resumo

O relatório resume a experiência de 2021 na disciplina Família e Tecnologia, que integra o currículo do Bacharelado em Aconselhamento Familiar da Universidade Austral da Argentina, no terceiro ano do curso. É um espaço a distância, desenvolvido no campus virtual da universidade, na plataforma Moodle 3.9, com carga horária intensiva de 60 horas por mês.

Como centro da proposta, o dispositivo TUYA - trajetórias ubíquas e autorreguladas - compõe uma abordagem inovadora e disruptiva, contrastando com as abordagens usuais dos espaços educacionais online. O objetivo é promover a autorregulação dos alunos, através de uma abordagem do objeto de estudo e dos conteúdos específicos da disciplina com base nos seus interesses particulares, em que a autogestão do tempo se apresenta como um elemento central. Isso se dá a partir de um acesso ubíquo, não só do corpo discente ao campus, mas também dos participantes imersos na própria dinâmica da sala de aula virtual.

$O$ resultado da experiência traduz-se em aprendizagens significativas, tanto para alunos como para professores, pois evidenciam as produções, mas também as marcas dos processos reflexivos promovidos durante a visita e aprofundados no encerramento do curso.

Palavras-chave: educação online, aprendizagem ubíqua, personalização, autorregulação, educação baseada em competências.

\section{Producir conocimientos, desarrollar habilidades, asumir actitudes ${ }^{1}$}

Los objetivos de la asignatura se inscriben en un enfoque basado en competencias, concepto que no solo implica conocimientos, sino también habilidades y actitudes. Según Nodine (2016), al menos tres elementos son necesarios para el desarrollo en educación superior de proyectos por competencias:

- Objetivos de aprendizaje

Establecer claramente qué se entiende por "competencia" en cada área temática y de aprendizaje.

\footnotetext{
${ }_{1}$ El presente artículo es un informe de cátedra de la asignatura Familia y Tecnología 2021. Mi agradecimiento a las profesoras Sonia Calamante y Romina Silvetti, compañeras de aventuras académicas con quienes integramos un maravilloso equipo docente. Ellas hacen posible esta experiencia educativa, a cada paso y en cada detalle.
} 
Austral Comunicación

Volumen 10, número 2 (Diciembre de 2021): e9-e23

ISSN (I) 2313-9129. ISSN (E) 2313-9137

- Evaluación de resultados

Identificar cómo los estudiantes pueden demostrar tal competencia a través de actividades y desempeños.

- Modelos flexibles y educación personalizada

Migrar de la formación con modelos y métodos estandarizados al uso de modelos y métodos flexibles, basados en las necesidades particulares de los estudiantes.

Estos tres componentes definen nuestra propuesta, concebida como un sistema dinámico que se retroalimenta y busca la mejora con cada nueva edición de la asignatura.

Con relación al primero de los ítems, nuestros objetivos están claramente explicitados y comunicados a nivel general y en cada una de las actividades, que cuentan con matrices de valoración puestas a disposición de los estudiantes junto con la correspondiente consigna.

La evaluación de los aprendizajes es formativa, con énfasis en el proceso, y a esto se le suma una valoración global de la materia concretada al finalizar, la cual convoca a todos los actores intervinientes. Finalmente, la flexibilidad y la personalización están dadas desde el mismo diseño tecnopedagógico -como se verá seguidamente-, presentándose como atributos esenciales de la propuesta formativa.

Por otra parte, se apunta a englobar los tres componentes del concepto "competencia" en cada momento de la intervención educativa. Tales componentes, como lo adelantamos, son: conocimientos, habilidades y actitudes (Yáñiz, 2008), por lo que se promueven adquisiciones y desarrollos de los estudiantes en cada uno de estos campos.

En el plano del conocimiento, la cátedra se propone concretar una aproximación al concepto "tecnología" desde una visión abarcativa, multiperspectiva, que contribuya a expandir nuestra conciencia sobre nuestra relación con ella y su presencia en la vida humana, favoreciendo la comprensión de la relevancia de los medios digitales en la cotidianidad familiar, a partir de la identificación de sus dinámicas en estudios recientes que recogen evidencia empírica y elaboran recomendaciones.

Para el despliegue de habilidades, el espacio impulsa a que los participantes experimenten el uso de redes sociales con vistas a entender su lógica y posibilidades, adquiriendo herramientas para la intervención socioeducativa y terapéutica desde la orientación familiar en cuestiones relativas al vínculo de las personas con las tecnologías y las plataformas sociodigitales.

Con vistas a la generación de actitudes, los objetivos de la cátedra apuntan a que los estudiantes trabajen colaborativamente y se constituyan en usuarios competentes, produciendo contenido, interactuando y dándoles feedback a sus pares.

Se pretende que profundicen su capacidad de autorregulación de los aprendizajes mediante el trazado de una trayectoria personal que les permita gestionar sus tiempos 
Mariángeles Castro Sánchez

El dispositivo TUYA como estrategia de personalización en educación en línea

y enfocarse en los puntos de interés propios, que extraigan conceptos del marco referencial y puedan relacionarlos, extrapolarlos y aplicarlos a situaciones concretas.

Adicionalmente, desde la perspectiva comunicacional, se aspira a que desarrollen capacidades de oralidad y escucha, logrando un intercambio conversacional satisfactorio con sus pares sobre temas específicos.

\section{Otra mirada de las tecnologías}

En Familia y Tecnología asumimos una mirada amplia de las tecnologías a partir de una reflexión sobre las dinámicas tecnosociales que se encuadran hoy en el ámbito familiar, con especial foco en las tecnologías digitales. Este encuadre apunta a brindar herramientas para que los egresados de Orientación Familiar puedan intervenir positivamente a través de acciones de prevención y de prácticas de reconducción frente a situaciones críticas.

Lo cierto es que si visualizamos la imagen de una familia actual en una situación de la vida diaria y meditamos sobre ella unos instantes, nos daremos cuenta de que en esta representación no podemos prescindir de la tecnología. Desde una simple comunicación verbal o escrita hasta el complejo acto de leer una revista en soporte papel participan de una dimensión que categorizamos como humana: la dimensión tecnológica (Castro Sánchez, 2019a). En el escenario actual, los medios digitales se han integrado en la vida familiar y han introducido nuevas formas de comunicación que vinieron a convivir con las ya establecidas, configurando un sistema ecológico de medios.

La noción de que la tecnología es una dimensión humana equivale a decir que los seres humanos estamos tecnológicamente constituidos (Thomas y Buch, 2013), que somos seres tecnológicos. Somos seres sociales y las sociedades están tecnológicamente configuradas. Surge aquí una noción abierta de tecnología, no reduccionista de lo tecnológico a lo artefactual. Y siguiendo nuestra línea de razonamiento, observamos que las tecnologías son sociales, por lo que afirmamos que las tecnologías son humanas (Castro Sánchez, 2019a).

La dimensión tecnológica atraviesa la existencia personal y permea todos los ámbitos de la vida en sociedad. Porque, como adelanta Garro-Gil (2017), desde una antropología relacional lo humano no se reduce únicamente a las personas, sino que también "las estructuras, los sistemas y las organizaciones sociales están llamados a ser cada vez más humanos, puesto que humano puede ser, en potencia, todo aquello que está en relación" (p. 637). Para Donati (2011), el hombre es un ser-con-otro, un ser-en-relación, y su personalidad es interpersonalidad. La apertura a los demás es parte constitutiva del ser personal. Justamente en este ser social del humano está implícito su ser tecnológico (Castro Sánchez, 2019a). 
Austral Comunicación

Volumen 10, número 2 (Diciembre de 2021): e9-e23 ISSN (I) 2313-9129. ISSN (E) 2313-9137

No obstante, la capacidad de transformación no es algo intrínseco a las tecnologías, sino a la propia relación de esta con las personas, puesto que coevolucionamos. La mirada debe estar puesta en lo interaccional, porque no es ni positivo ni negativo en sí, pero tampoco es neutral. Es relacional.

Livingstone y Helsper (2008) advierten que la agenda de la investigación ya no se centra en la relación de los niños con internet como medio, sino que, más profundamente, refiere a la relación de los niños con el mundo mediada por internet. Esto significa que todos y cada uno de los componentes del modelo socioecológico -familia, educadores, instituciones, cultura- debemos reconfigurarnos en la era digital, ya que no significamos lo que significábamos en contextos analógicos ni funcionamos del modo en el que lo hacíamos antes (Castro Sánchez, 2019a).

Esta es la mirada que atraviesa la fijación de objetivos y el desarrollo temático de Familia y Tecnología, que se detalla en la Figura 1.

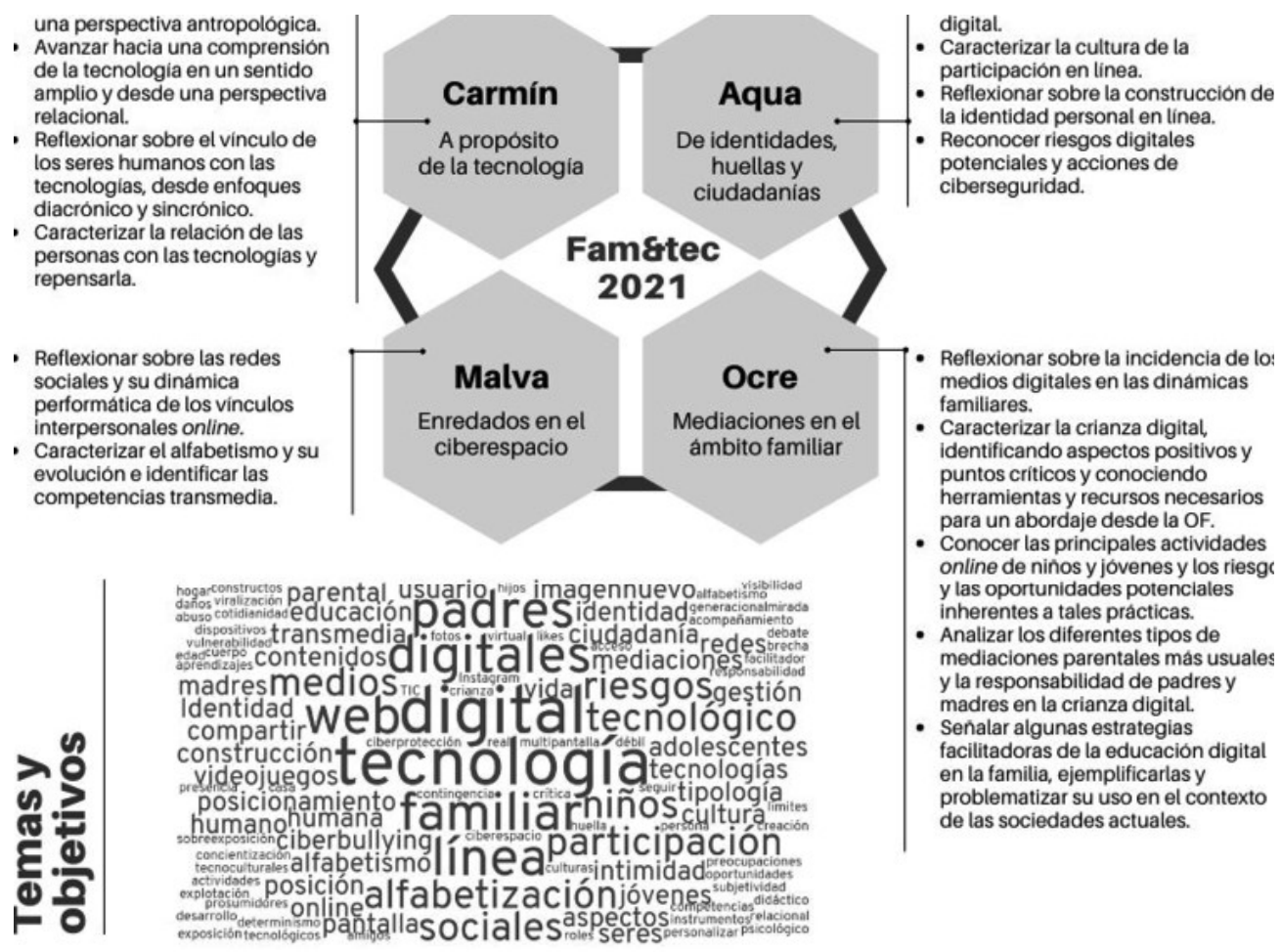

Figura 1. Objetivos y desarrollo temático de Familia y Tecnología 2021. Fuente: elaboración propia a partir del programa 2021 de Familia y Tecnología.

Situados en esta perspectiva relacional, la cátedra compone una metáfora de nuestras prácticas como usuarios de plataformas sociodigitales, aplicaciones y de la propia 
Mariángeles Castro Sánchez

El dispositivo TUYA como estrategia de personalización en educación en línea

web, reproduciendo la lógica del click in y el click out, la navegación fragmentada y el on demand. El diseño tecnopedagógico busca reproducir esta experiencia, tal como se lo explica en los apartados siguientes.

\section{Diseño tecnopedagógico}

Siguiendo a Henry y Meadows (2007), nos propusimos avanzar hacia una propuesta diferente a partir de un modelo pedagógico que monta un entorno de aprendizaje efectivo y atractivo, el cual genera experiencias significativas y alcanza un elevado nivel de satisfacción en los participantes. Uno de los objetivos primordiales fue lograr que el estudiantado se focalice en el curso y que el entorno tecnológico se vuelva transparente durante el proceso. Asimismo, la meta a alcanzar en el período de diseño del curso fue que se involucre activamente en su propio aprendizaje, motivarlo e interpelarlo dentro de un ambiente que sirva de andamiaje e inspiración.

Esta etapa fue un momento de creatividad absoluta. En tal sentido, pusimos en práctica la sentencia de Maggio (2018) respecto a que las experiencias de aprendizaje deben estar solo limitadas por la imaginación, por la capacidad de soñarlas, ya que siempre pueden ir más allá, abarcar actividades muy diversas y situarse en la frontera de lo conocido y lo experimental.

Sabemos que el diseño de una intervención formativa en línea atiende a una multiplicidad de factores que condicionan el diseño tecnopedagógico, y que este se concibe como un espacio que dialoga con otros y con el afuera a través de actividades y recursos que involucran plataformas externas (Schwartzman et al., 2014). En consonancia con ello, Familia y Tecnología compone un entorno flexible y poroso que nos mueve a explorar otros territorios de la web.

El corpus textual seleccionado sirve de hilo conductor de un recorrido que se aborda desde un enfoque personalizado y conjuga la lectura de la bibliografía, el acceso a materiales hipermediales y la ejecución de actividades para favorecer la apropiación de los contenidos y el logro de aprendizajes significativos. Lo esencial reside en su carácter innovador, porque este es un rasgo relevante de su identidad en tanto vía que nos conduce hacia algo nuevo. Si bien entendemos que cada dispositivo tecnopedagógico es único "porque expresa las necesidades y condiciones de un proyecto pedagógico particular" (Schwartzman et al., 2014, p. 46), buscamos construir esa unicidad y logramos consolidarla.

\section{El dispositivo TUYA}

Como lo expresamos precedentemente, la asignatura se plantea como un sistema de contenidos, recursos y actividades susceptibles de ser abordados en el orden y con 
Austral @municación

Volumen 10, número 2 (Diciembre de 2021): e9-e23 ISSN (I) 2313-9129. ISSN (E) 2313-9137

los tiempos que cada estudiante define a partir de sus intereses particulares y sus saberes previos, sin la necesidad de acudir a una secuencia prefijada.

Así, el espacio aula se estructura en un dispositivo denominado TUYA (trayectorias ubicuas y autorreguladas), que admite que los participantes elijan por dónde comenzar y qué orden seguir, completando un itinerario a través de cuatro estaciones nombradas con colores: carmín, aqua, ocre y malva. Esta denominación se propone romper con cualquier sugerencia de orden preestablecido, dejando al estudiantado al frente de procesos individuales de toma de decisiones y de trazado de itinerarios personales.

Tal como lo destaca Albarello (2019), en el aprendizaje ubicuo los aprendices crean el conocimiento de forma activa y en esta creación dan cuenta de su bagaje previo, sus saberes, experiencias y visiones. Esto es lo nuclear de la propuesta, más allá de la ubicación física que puedan tener.

Una presentación interactiva elaborada en Genial.ly -e incrustada en Moodle- es el punto de partida hacia los múltiples destinos programáticos, que cuentan con actividades como nodos de conexión entre los participantes (Figura 2).

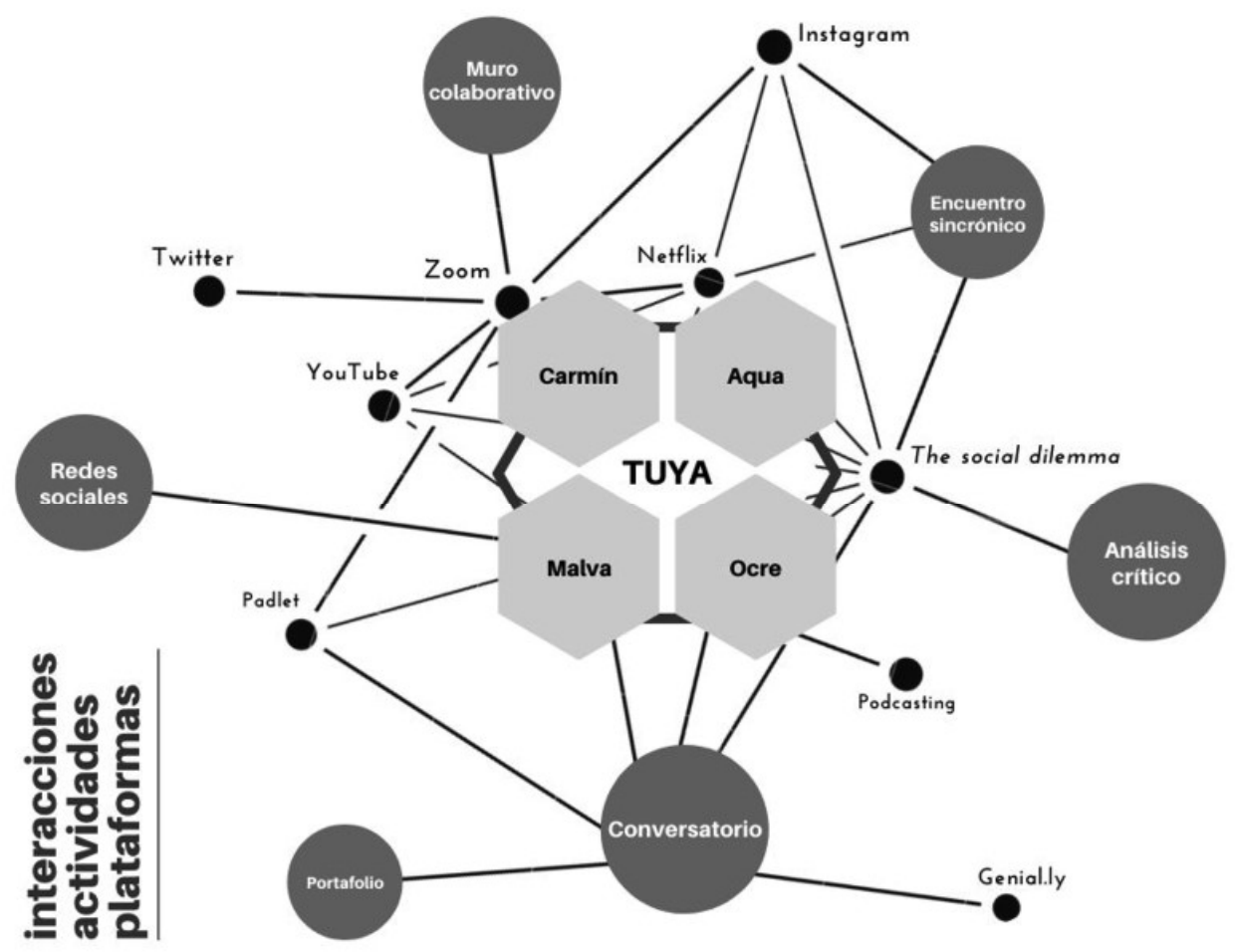

Figura 2. Las actividades y plataformas como nodos de interacción. Fuente: elaboración propia. En el centro de la propuesta formativa se sitúa el dispositivo TUYA. Los itinerarios se trazan en múltiples direcciones, generando una red de sentidos en la que las actividades y plataformas operan como nodos de interacción. 
Mariángeles Castro Sánchez

El dispositivo TUYA como estrategia de personalización en educación en línea

\section{Las actividades, core de la propuesta formativa}

En educación en línea, las actividades son la clave de la propuesta formativa. Henry y Meadows (2008) señalan que en el aula virtual el contenido es un verbo y que las propuestas deben migrar del delivery de información a la creación de experiencias de aprendizaje centradas en tareas. La clave es hacer para -haciendo- alcanzar los objetivos de aprendizaje previstos.

En el mismo sentido, Schwartzman et al. (2014) coinciden en que es necesario generar condiciones para que los estudiantes pongan en juego los contenidos y recursos, lo cual se da a través de las actividades. Por su parte, Pardo Kuklinski y Cobo (2020) invitan a pensar los contenidos como una narrativa transmedia expandida que abarque momentos sincrónicos y asincrónicos: contenidos de consumo pasivo (broadcasting, uno a muchos) y otros que sitúen al estudiante en el rol de prosumidor, usuario y consumidor a la vez. El codiseño de contenidos es una alternativa que los autores remarcan y que se llevó a término en esta edición de la asignatura, a través de muros, podcasts e interacciones en redes sociales.

Las actividades de aprendizaje fueron las siguientes (Figura 2):

- Creación de perfiles y participación en redes sociales -Instagram y Twitter-, con el objeto de exponer ideas, plantear interrogantes y argumentaciones sobre los tópicos de debate y avanzar en la producción de conclusiones a partir de los intercambios mantenidos.

- Construcción de un portafolio de actuación en redes en el que se incluyen los posteos, discusiones y aportaciones producidos.

- Elaboración de un análisis crítico de The social dilemma (Orlowsky, 2020) -un híbrido documental-drama disponible en Netflix-, dando cuenta del recorrido personal por el corpus bibliográfico y weblográfico de la asignatura. El propósito está en poner en práctica habilidades de argumentación, destacando un punto de coincidencia y otro de divergencia con la perspectiva adoptada por los realizadores y justificando ambas posturas. Se apunta aquí a la extrapolación de conceptos aprendidos y su aplicación a la fundamentación de determinados posicionamientos.

- Producción en duplas de un conversatorio en formato podcast, con el fin de desarrollar la oralidad y acrecentar habilidades para el diálogo y el trabajo colaborativo.

- Participación en masterclass sincrónica, con la presencia de Francisco Albarello como profesor invitado.

- Reflexión sobre la experiencia y puesta en común en un Padlet colaborativo titulado: ¿Cómo la estoy llevando? Un ejercicio metacognitivo cuyas marcas se registran en el espacio común y se funden con las de los restantes participantes. 


\section{Austral Omunicación \\ Volumen 10, número 2 (Diciembre de 2021): e9-e23 ISSN (I) 2313-9129. ISSN (E) 2313-9137}

\section{Evaluar para aprender}

Un diseño de evaluación por competencias implica que el estudiante se ubique frente al reto de la integración y la movilización de conocimientos, destrezas y actitudes para poder afrontar y resolver un problema dado (Díaz Barriga y Pérez Rendon, 2010). En Familia y Tecnología, el enfoque por competencias se concreta en una transversalidad que enfatiza el proceso y está centrada en las actividades. Por ello, se emplean matrices de valoración de desempeño en las diferentes dinámicas propuestas, que los estudiantes reciben junto a la consigna de cada actividad para así poder conocer qué criterios serán tenidos en cuenta en el momento de valorar sus producciones.

Cobo (2016) nos recuerda que las evaluaciones formativas actúan como indicador de progreso en los aprendizajes y que esto, a su vez, correlaciona con el nivel de eficacia de la enseñanza. Brindan información durante el desarrollo, posibilitando que se identifiquen áreas de mejora y se produzca una orientación longitudinal y un seguimiento de resultados. Este enfoque presta atención al estudiante y su proceso y brinda retroalimentación de modo constante. Las propuestas personalizadas nos llevan a comprender de manera profunda el trabajo de los estudiantes y a acompañarlos en sus trayectorias y procesos. Esto es tan movilizador que colocar una calificación numérica "nos termina pareciendo un acto banal" (Maggio, 2018, p. 115).

Como lo advierte esta autora, "si la enseñanza puede ser un acto de invención, la evaluación debería ser la mejor expresión de ese proyecto" (Maggio, 2018, p. 93). Se trata aquí -y así lo asumimos en la cátedra- de que los estudiantes se apropien no solo de los contenidos, sino fundamentalmente del proceso de aprender, que asuman responsabilidades e incrementen su autonomía (Anijovich y González, 2011). Se trata de aprender a aprender y de evaluar para seguir aprendiendo.

\section{Percepciones del estudiantado}

En busca de mejorar progresivamente la propuesta, se les administraron cuestionarios de valoración a los diferentes actores implicados: estudiantes, docentes y equipo de gestión de la carrera. Seguidamente, presentamos los resultados obtenidos del estudiantado.

La respuesta, en todos los casos, fue anónima y obligatoria, pues estuvo vinculada a la posibilidad de entrega del último de los desempeños. Se realizó, entonces, un muestreo censal en el que la muestra coincide con la población estudiada (Hayes, 1999), aplicando un cuestionario de 9 posiciones, de las cuales 5 son abiertas y 4 están cerradas a opciones.

En la Figura 3 observamos que, en su mayoría, los encuestados valoraron positivamente la cursada. Sobre un total de 48 participantes, 44 tuvieron una experiencia favorable, 4 la calificaron como neutral y ninguno como desfavorable. 
Mariángeles Castro Sánchez

El dispositivo TUYA como estrategia de personalización en educación en línea

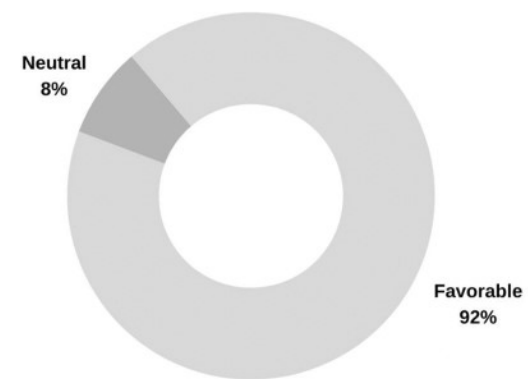

Figura 3. Balance de cursada para el estudiantado. Fuente: elaboración propia.

Sobre la posición abierta $\mathrm{N}^{\circ} 2$, que solicita que se fundamente el balance de la cursada, se realiza una codificación asistida por el software ATLAS.ti, emergiendo las categorías de análisis que se detallan en la Tabla 1 (con base en las citas) y que se ponen en relación en la Figura 4.

El contenido y las actividades, así como la propuesta de trabajo colaborativo, son vistas como fuente de enriquecimiento y motivación. Este último punto está reforzado por la dimensión de actualidad y la posibilidad de gestionar los propios tiempos de dedicación a la cursada, que son valorados como oportunidades. Esto se enlaza, a su vez,

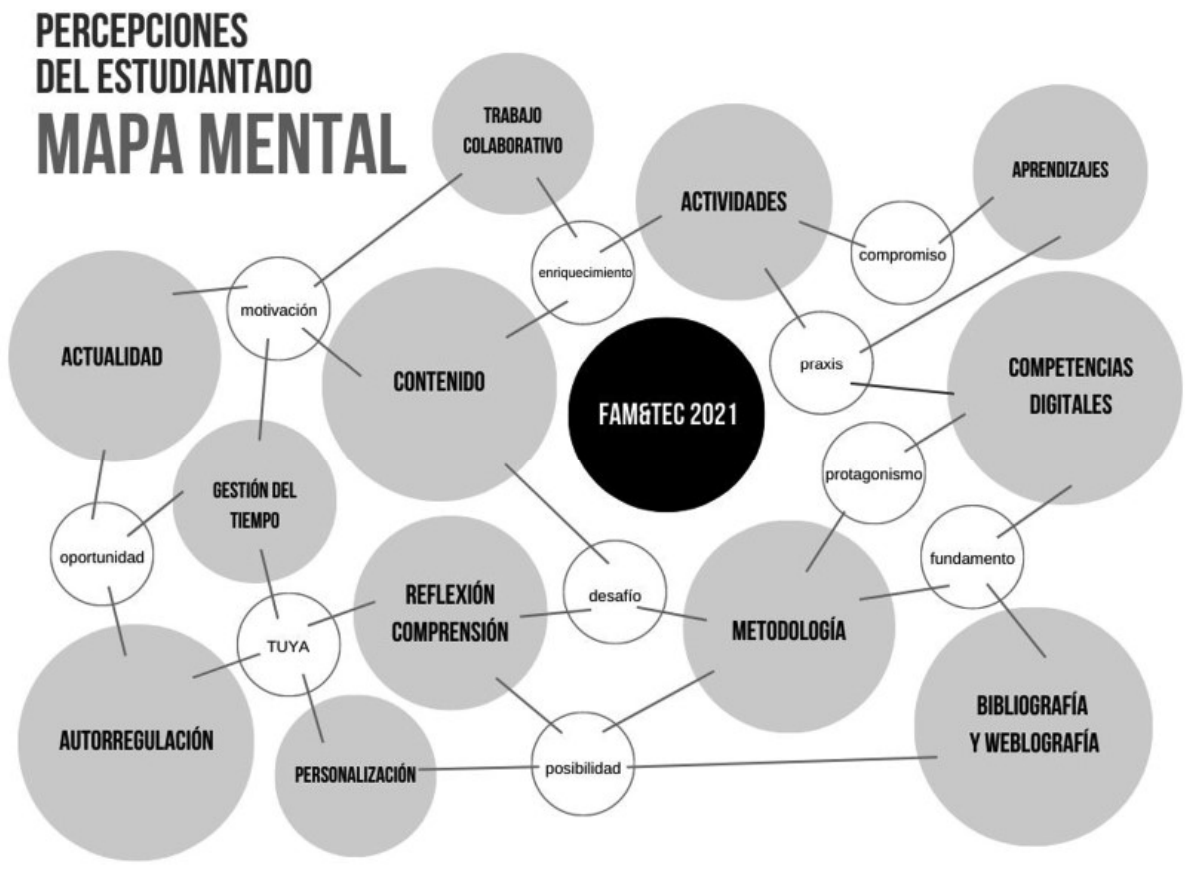

Figura 4. Percepciones del estudiantado. Mapa mental. Fuente: elaboración propia. En el núcleo está la asignatura, rodeada de las categorías emergentes del microanálisis de los datos recolectados. El tamaño de las figuras es proporcional a la cantidad de citas asociadas. Los círculos pequeños funcionan como conectores relacionales. 
Austral Comunicación

Volumen 10, número 2 (Diciembre de 2021): e9-e23 ISSN (I) 2313-9129. ISSN (E) 2313-9137

Tabla 1. Categorías emergentes y citas asociadas

\begin{tabular}{|c|c|c|}
\hline Categorías & Citas & Destacadas \\
\hline Contenido & 7 & $\begin{array}{l}\text { "Muy buen contenido y organización de las actividades". } \\
\text { "Una materia con conocimientos necesarios que brinda una } \\
\text { mirada innovadora en la cursada". }\end{array}$ \\
\hline \multirow[t]{2}{*}{ Competencias digitales } & 7 & $\begin{array}{l}\text { "Me ayudó a incursionar en nuevas tecnologías, como } \\
\text { Instagram y Twitter, que son fundamentales en la } \\
\text { comunicación del siglo XXI. Amplió mi mirada sobre lo } \\
\text { tecnológico". }\end{array}$ \\
\hline & & $\begin{array}{l}\text { "Me impulsó a romper mis propias limitaciones con el uso de } \\
\text { las redes sociales. El desafío fue productivo y educativo, hoy } \\
\text { tengo más herramientas". }\end{array}$ \\
\hline \multirow[t]{2}{*}{ Biblio y weblografía } & 7 & "Material interesante y práctico". \\
\hline & & "Muy buen material audiovisual y material de lectura". \\
\hline Autorregulación & 7 & $\begin{array}{l}\text { "Autorregular las lecturas y las actividades genera un } \\
\text { compromiso distinto ante el aprendizaje". } \\
\text { "Me parece favorable poder gestionar las actividades y } \\
\text { tiempos de estudio". }\end{array}$ \\
\hline Metodología & 6 & $\begin{array}{l}\text { "Me gustó mucho el planteo de la materia: cursarla con } \\
\text { libertad y en base a la propia organización". } \\
\text { "Un gran desafío la forma en que está dada la materia, } \\
\text { totalmente desestructurada". }\end{array}$ \\
\hline Actualidad & 6 & $\begin{array}{l}\text { "No sabía que éramos seres tec nológicos... Encontré otra } \\
\text { mirada sobre los medios digitales que me nutrió mucho". } \\
\text { "[...] Muy actual, muy interesante aprender sobre el uso } \\
\text { responsable de las redes". }\end{array}$ \\
\hline \multirow[t]{2}{*}{ Reflexión comprensión } & 5 & $\begin{array}{l}\text { "La tecnología no tenía una presencia gravitante en mi vida. } \\
\text { Simplemente era una herramienta a mi disposición, si bien es } \\
\text { verdad que le reconocía la influencia... Hoy, con la perspectiva } \\
\text { relacional, redimensioné esta mirada y pude ubicarla en su } \\
\text { punto justo". }\end{array}$ \\
\hline & & $\begin{array}{l}\text { "Generó un espacio de reflexión sobre temas que } \\
\text { habitualmente no pienso con tanta profundidad". }\end{array}$ \\
\hline Actividades & 5 & $\begin{array}{l}\text { "Las lecturas, actividades y el encuentro sincrónico han sido } \\
\text { muy enriquecedores". }\end{array}$ \\
\hline Aprendizajes & 4 & $\begin{array}{l}\text { "Aprendí muchísimo, me sorprendió todo lo que no sé sobre } \\
\text { las redes". }\end{array}$ \\
\hline Gestión del tiempo & 3 & $\begin{array}{l}\text { "Me parece favorable poder gestionar las actividades y los } \\
\text { tiempos de estudio". }\end{array}$ \\
\hline Personalización & 2 & $\begin{array}{l}\text { "Me encantó la experiencia TUYA. Fue una cursada en la que } \\
\text { tuvimos que desplegar la autonomía y eso me resultó muy } \\
\text { favorable. Organizar el material de estudio, junto con los } \\
\text { recursos y las actividades por realizar". }\end{array}$ \\
\hline Colaboración entre pares & 2 & $\begin{array}{l}\text { "Nos fortaleció en el aprendizaje socializado y la ayuda entre } \\
\text { pares". }\end{array}$ \\
\hline
\end{tabular}

Fuente: elaboración propia a partir de la codificación efectuada con asistencia del software ATLAS.ti. 
Mariángeles Castro Sánchez

El dispositivo TUYA como estrategia de personalización en educación en línea

con el desarrollo de la autorregulación por medio del dispositivo TUYA, que evidencia un diseño favorecedor de la personalización educativa y la reflexión sobre las propias prácticas. Una metodología de enseñanza activa apunta a la adquisición de competencias y encuentra su fundamento en la bibliografía y la weblografía seleccionada por la cátedra para esta edición de la asignatura.

Coincidimos con Sangrá (2020) en que los modelos de educación en línea funcionan cuando se fundan en interacciones significativas entre los diferentes participantes. En este sentido, es mucho más que facilitar el acceso de determinados recursos o contenidos, ya que es precisamente la calidad de las interacciones la que define una propuesta educativa en línea. Así lo han estudiado Garrison et al. (2000) desde el modelo Community of Inquiry (CoI). Los autores destacan que el propósito de una experiencia educativa es su estructuración para el logro de resultados de aprendizaje definidos.

En la educación en línea, las interacciones deben estructurarse de modo sistemático. La formación de comunidad resulta esencial para el logro de objetivos; la interacción y la reflexión deben sostenerse en un proceso crítico andamiado y modelado. Las interacciones deben ser variadas, porque van más allá de lo social y del simple intercambio de información: abarcan combinaciones de interacción entre el contenido, los docentes y los estudiantes (Garrison y Cleveland-Innes, 2005). El modelo desarrollado por estos autores asume que los aprendizajes se concretan dentro de una comunidad, y para que puedan desarrollarse positivamente también deben tenerse presentes las esferas afectiva y emocional (Castro Sánchez, 2019b), porque parten de la intersección de tres presencias: la cognitiva, la docente y la social.

Dado que las interacciones multidireccionales que surgen en esta propuesta formativa constituyen un punto clave para el desarrollo de los aprendizajes, se realizó una valoración de este ítem particular, el cual arroja el resultado que muestra la Figura 5.

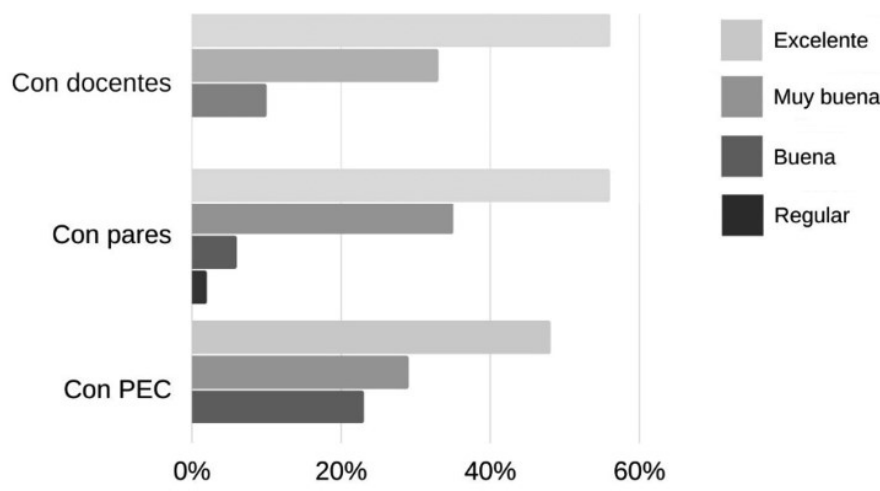

Figura 5. Valoración de las interacciones durante la cursada. Fuente: elaboración propia. La denominación $P E C$ refiere a profesor encargado de cohorte, rol docente responsable de realizar un seguimiento personalizado de cada estudiante de una cohorte a lo largo de un año académico. 
Austral @municación

Volumen 10, número 2 (Diciembre de 2021): e9-e23 ISSN (I) 2313-9129. ISSN (E) 2313-9137

En la última de las posiciones del cuestionario administrado se les pidió a los estudiantes que sintetizaran la experiencia global de aprendizaje en una sola palabra. La Figura 6 presenta en una nube los resultados obtenidos.

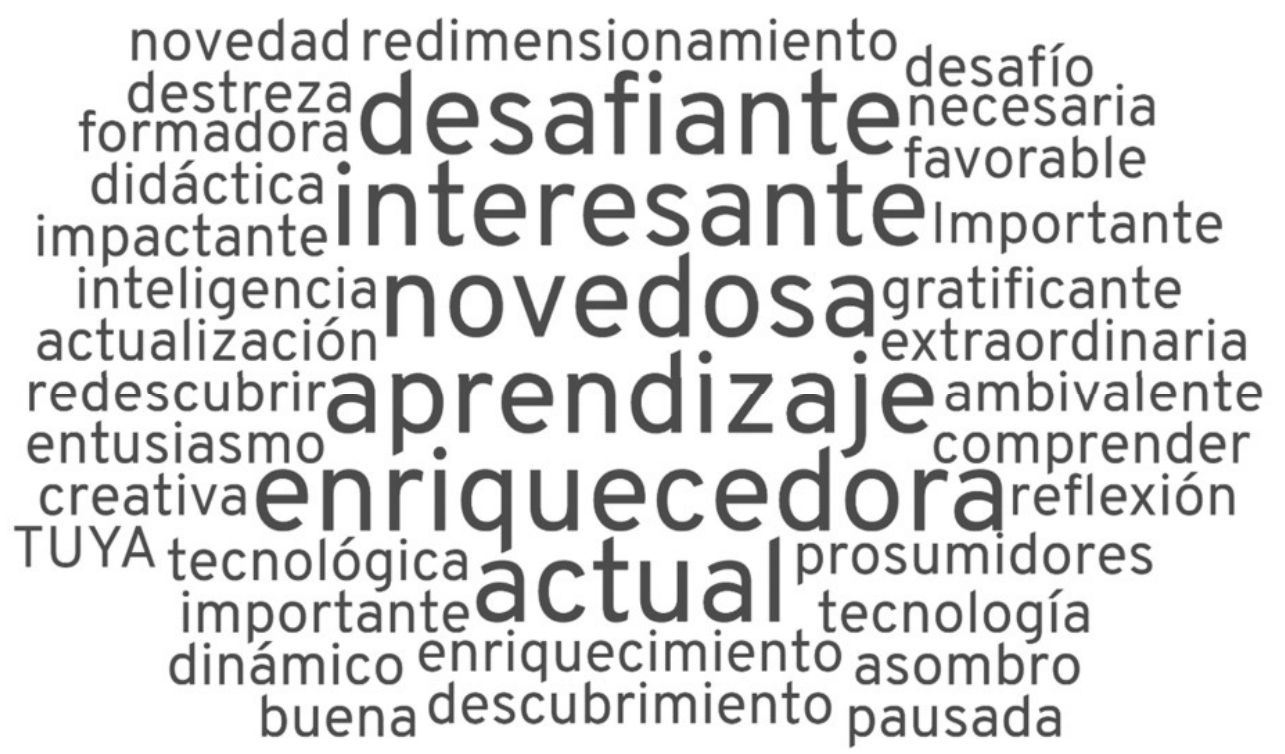

Figura 6. Síntesis de la experiencia en una sola palabra. Fuente: elaboración propia a partir de la sexta posición del cuestionario administrado al cierre de la cursada.

\section{Notas conclusivas}

Nuestra propuesta constituye una experiencia diferente que aspira a involucrar a los estudiantes en sus propios procesos de aprendizaje, de modo que conecten con sus saberes previos, descubran cuáles son sus intereses y planifiquen sus acciones en función de los objetivos previstos. Esta modalidad favorece la autonomía y la autorregulación, capacidades relevantes para la vida académica y también para el futuro desempeño profesional. Esto, lejos de dejarlos en soledad, refuerza la cercanía y el acompañamiento durante sus progresiones individuales hacia los nodos de encuentro.

De acuerdo con las manifestaciones de los participantes, podemos confirmar que cuando la propuesta suma y los aprendizajes se producen, esto se expresa y se valora positivamente.

Por otra parte, observamos que un modelo basado en competencias se conjuga muy bien con una educación en línea vivida dentro del paradigma de la personalización educativa. Ambos enfoques encuentran su correlato en una implicación activa de los participantes en sus aprendizajes y en la producción colaborativa de conocimiento. Estos componentes actúan como potenciadores de la experiencia y del nivel de motivación del conjunto. 
Mariángeles Castro Sánchez

El dispositivo TUYA como estrategia de personalización en educación en línea

El dispositivo TUYA constituye una estrategia válida que se nutre de las oportunidades que la plataforma Moodle ofrece y abre el espacio a la creatividad de los estudiantes en función de sus intereses personales.

Adicionalmente, remarcamos que la calidad de las interacciones define la propuesta, que estas son multidireccionales y que abarcan tres esferas: contenidos, pares y docentes. Estos últimos, a su vez, aprenden durante todo el recorrido y se ocupan de traducir lo incorporado en mejoras para próximas ediciones del curso.

De cara al futuro de Familia y Tecnología, proyectamos continuar indagando en nuevas estrategias de educación en línea personalizada, en la seguridad que la experimentación pedagógica en educación superior nos abre a formas diversas de articular el currículo con el mundo del trabajo. Un mundo que reclama una formación integral, expresada en el desarrollo de competencias transversales y específicas, en el despliegue de un saber hacer, un querer hacer y un poder hacer que confluyen como resultado de procesos formativos personalizados. Esta certeza está en el centro de nuestra intervención educativa.

\section{Referencias}

Albarello, F. (2019). Lectura transmedia. Leer, escribir, conversar en el ecosistema de pantallas. Ampersand.

Anijovich, R. y González, C. (2011). Evaluar para aprender. Conceptos e instrumentos. Aique.

Castro Sánchez, M. (2019a). Familias 4.0: lo virtual es real. VI Congreso Internacional de las Relaciones Interpersonales: Desarrollo humano en tiempos de la (re) evolución 4.0. Centro de Estudio de las Relaciones Interpersonales, Universidad Austral, Buenos Aires.

Castro Sánchez, M. (2019b). Vínculo pedagógico y compromiso académico en la universidad. Estudio de caso desde la perspectiva de los estudiantes (Tesis de Maestría en Dirección de Instituciones Educativas). Escuela de Educación, Universidad Austral, Buenos Aires.

Cobo, C. (2016).La innovación pendiente. Reflexiones (y provocaciones) sobre educación, tecnología y conocimiento. Debate.

Díaz Barriga Arceo, F. y Pérez Rendon, M. M. (2010). El portafolio docente a escrutinio: sus posibilidades y restricciones en la formación y evaluación del profesorado. Observar, 4, 6-27.

Donati, P. (2011). Relational Sociology: A New Paradigm for the Social Sciences. Routledge.

Garrison, R., Anderson, T. y Archer, W. (2000). Critical Inquiry in a Text-Based 
Environment: Computer Conferencing in Higher Education. The Internet and Higher Education, 2(2-3), 87-105.

Garrison, R. y Cleveland-Innes, M. (2005). Facilitating Cognitive Presence in Online Learning: Interaction Is Not Enough. The American Journal of Distance Education, 19(3), 133-148.

Garro-Gil, N. (2017). Relación, razón relacional y reflexividad: tres conceptos fundamentales de la sociología relacional. Revista Mexicana de Sociología, 79(3), 633-660.

Hayes, B. (1999). Cómo medir la satisfacción del cliente: diseño de encuestas, uso y métodos de análisis estadístico. Oxford University Press.

Henry, J. y Meadows, J. (2008). An absolutely riveting online course: Nine principles for excellence in web-based teaching. Canadian Journal of Learning and Technology, 34(1). https://www.learntechlib.org/p/43238/.

Livingstone, S. y Helsper, E. (2008). Parental Mediation of Children's Internet Use. Journal of Broadcasting \& Electronic Media, 52(4), 581-599. https://doi. org/10.1080/08838150802437396, 581-599.

Maggio, M. (2018). Reinventar la clase en la universidad. Paidós.

Nodine, T. R. (2016). How did we get here? A brief history of competency-based higher education in the United States. The Journal of Competency-Based Education, 1(1), 5-11. http://dx.doi.org/10.1002/cbe2.1004.

Orlowsky, J. (Director). (2020). The social dilemma [Película]. Exposure Labs.

Pardo Kuklinski, H.y Cobo, C. (2020). Expandir la universidad más allá de la enseñanza remota de emergencia. Ideas hacia un modelo híbrido post-pandemia. Outliers School.

Sangrá, A. (Coord). (2020). Decálogo para la mejora de la docencia online. Propuestas para educar en contextos presenciales discontinuos. Editorial UOC.

Schwartzman, G., Tarasow, F. y Trech, M. (2014). Dispositivos tecnopedagógicos para enseñar: el diseño en la Educación en Línea. En Schwartzman, G., Tarasow, F. y Trech, M. (Comps.), De la Educación a Distancia a la Educación en Línea. Aportes a un campo en construcción (pp. 37-62). Homo Sapiens.

Thomas, H. y Buch, A. (2013). Actos, actores y artefactos: Sociología de la tecnología. Universidad Nacional de Quilmes.

Yáñiz, C. (2008). Las competencias en el currículo universitario: implicaciones para diseñar el aprendizaje y para la formación del profesorado. Revista de Docencia Universitaria, 4(2), 31-39. 